Ilchmann, Achim; Owens, David H.:

Exponential Stabilization using Piecewise Constant Gain Adaptation

Aus:

Proceedings of the 30th IEEE Conference on Decision and Control : 11 - 13 December 1991, Brighton, England / IEEE Control Systems Society; Vol 1. - New York : IEEE, 1991, S. 83-84 DOI: $\underline{10.1109 / C D C .1991 .261260}$ 


\title{
Exponential Stabilization using Piecewise Constant Gain Adaptation
}

\author{
Achim Ilchmann and David H. Owens \\ Centre for Systems and Control Engineering \\ School of Engineering, University of Exeter \\ North Park Road \\ Exeter, Devon EX4 4QF, U.K.
}

\begin{abstract}
An adaptive stabilizer for the class of SISO minimum phase systems with known sign is introduced. It extends the work of Willems and Byrnes but uses a 'threshold' based piecewise constant gain adaptation. This provides an exponential decaying state and moreover the set of thresholds which stabilize the terminal system exponentially are dense.
\end{abstract}

\section{Introduction}

In recent years attention has been focussed on non identifier based adaptive stabilization of SISO linear systems of the form

$$
\dot{x}(t)=A x(t)+b u(t), y(t)=c x(t), x(0) \in \mathbb{R}^{n}
$$

where the state space dimension $n$ is unknown, it is only assumed that the $\operatorname{sign}(c b)$ is known and the system is minimum phase, i.e.

(i) The transfer function has no zeros in $\overline{\mathbb{C}_{+}}$.

(ii) $(A, b, c)$ is stabilizable and detectable.

The simplest adaptive controller for this class of systems is due to Willems and Byrnes [5] and is based on the idea that the time-varying parameter $k(t)$ in the output feedback

$$
u(t)=-\operatorname{sgn}(c b) k(t) y(t)
$$

finds the appropriate gain level by adapting $k(\cdot)$ via the evolution law

$$
\dot{k}(t)=y(t)^{2}, \quad k(0)=k_{0} \in \mathbb{R} .
$$

For every initial data $x_{0}, k_{0}$ the closed loop system (1.1)-(1.3) guarantees that $\lim _{t \rightarrow \infty} x(t)=0$ and $\lim _{t \rightarrow \infty} k(t)=k_{\infty}$ exists.

Based on these ideas, in Section 2 we present an adaptive stabilizer which uses a piecewise constant gain $k(\cdot)$ adjusted via a sequence of thresholds. This provides the bonus of exponential decaying of the state. However the terminal system $\dot{x}=\left[A-k_{\infty} b c\right] x$ is not necessarily stable. In Section 3 we show that for every fixed initial condition $x_{0}$ the sequences of thresholds which lead to an exponentially stable terminal system are dense.

\section{Exponential decaying of the state}

Proposition 2.1: Let $\left\{T_{i}\right\}_{i \in \mathbb{N}} \subset \mathbb{R}_{+}$be a sequence of 'thresholds' which satisfies in $f_{i \in \mathbb{N}}\left\{T_{i+1}-T_{i}\right\}>0$. Then the adaptive controller

$$
\left.\begin{array}{l}
u(t)=-k(t) y(t) \\
\dot{s}(t)=y(t)^{2}, \quad s(0)=T_{1} \\
k(t)=T_{i} \text { for } \quad s(t) \in\left[T_{i}, T_{i+1}\right)
\end{array}\right\}
$$

applied to any minimum phase system of the form (1.1) with $c b>0$ yields a closed loop system with

(i) The solution $(x, s)$ of $(1.1),(2.1)$ exists for all $t \geq 0$.

(ii) $x(t)$ tends to zero exponentially.

(iii) $\lim _{t \rightarrow \infty} s(t)=s_{\infty}$ exists.

(iv) For some $j \in \mathbb{N}$ and $t^{*}>0, k(t)=T_{j}$ for all $t \geq t^{*}$.

Proof: (a) Since the right hand side of the closed loop system is discontinuous in $x$ existence and uniqueness of the solution as well as finite escape time has to be clarified. This can be done similar to [1] and considering the following steps on finite intervals first. We omit it for brevity and assume that $(x, s)$ exists on $\mathrm{IR}_{+}$.

(b) It has been proved in [2] that the minimum phase property implies the inequality

$$
\frac{1}{2} y(t)^{2} \leq M+M \int_{t_{0}}^{t} y(s)^{2} d s+c b \int_{t_{0}}^{t} u(s) y(s) d s
$$

for some $M=M\left(x_{0}\right)>0$ and all $t \geq t_{0}, t_{0} \geq 0$. Inserting the feedback into (2.2) yields

$$
\frac{1}{2} y(t)^{2} \leq M+\int_{t_{0}}^{t}[M-c b k(s)] y(s)^{2} d s .
$$

If $k(\cdot) \notin L_{\infty}$ then $y(\cdot) \notin L_{2}$ and the right hand side of (2.3) goes to $-\infty$ as $t$ tends to $\infty$. This contradicts the positivity of the left hand side and proves boundedness of $k(t)$.

(c) $k(\cdot) \in L_{\infty}$ yields $y(\cdot) \in L_{2}$ and a well know argument gives $\lim _{t \rightarrow \infty} x(t)=0$, see [5]. This proves (iii) and (iv).

(d) Using the notation of (iv) it follows that $x(t)$ satisfies

$$
\dot{x}(t)=\left[A-T_{j} b c\right] x(t) \quad, \quad \text { for all } t \geq t^{*} .
$$

Since the terminal system is linear time-invariant and detectable, every mode which is not exponentially stable would contradict the boundedness of $s(\cdot)$. Therefore (ii) is proved.

\section{Remark 2.2:}

(i) In case of unknown sign of the high frequency gain $c b$ the same result holds true if the control strategy is modified by using the concept of switching functions due to Nussbaum [4], see also [5].

(ii) Proposition 2.1 can be extended to the class of adaptive controllers which use piecewise constant gain adaptation. The essential ingredient is that after a finite time the closed loop system $\dot{x}=[A-k b c] x$ is a time invariant linear system. 
3 Exponential stability of the terminal system

In Section 2 we have shown that the adaptive stabilizer (2.1) ensures an exponential decaying state trajectory corresponding to the initial condition $x_{0} \in \mathbb{R}^{n}$. However the terminal system $\dot{x}=\left[A-T_{j} b c\right] x$ must not be exponentially stable, the trajectory may lie in a stable subspace.

In this section we shall show that for each fixed initial condition $x_{0} \in \mathbb{R}^{n}$ the set of thresholds which achieve an exponentially stable terminal system is dense in the set of all admissible thresholds.

Proposition 3.1: Under the assumptions of Proposition 2.1 for every fixed system (1.1) and every $\varepsilon>$ 0 there exists an $N \in \mathbb{N}$ and $T_{1}^{\prime}, \ldots, T_{N}^{\prime}$ such that $\left|T_{i}-T_{i}^{\prime}\right|<\varepsilon$ for all $i \in \underline{N}$ and the switching sequence $T_{1}^{\prime}, \ldots, T_{N}^{\prime}, T_{N+1}, \ldots$ used for the adaptive stabilizer yields an exponentially stable terminal system, i. e. $\sigma\left(A-T_{N}^{\prime} b c\right) \subset \mathbb{C}_{-}, \quad x_{c} \neq 0,(c, A)$ obser.able.

Proof: Using the notation

$A_{\varepsilon}:=A-(k+\varepsilon) b c$

$p_{\varepsilon}(s):=\operatorname{det}\left(s I_{n}-A_{\varepsilon}\right), \quad z(s):=c \cdot \operatorname{adj}\left(s I_{n}-A\right) \cdot b$

it follows that

$$
p_{\varepsilon}(s)=p_{0}(s)+\varepsilon z(s) \text { for all } \varepsilon \in \mathbb{I R} \text {. }
$$

We proceed in several steps.

(a) All eigenvalues of $A_{\varepsilon}$ in $\overline{\mathbb{C}_{+}}$are moving if $\varepsilon$ moves. Suppose the contrary, i. e. for some $s_{0} \in \overline{\mathbb{C}_{+}}$ const $\theta \equiv p_{0}\left(s_{0}\right)+\varepsilon z\left(s_{0}\right)$ locally in $\varepsilon$ around 0 .

This is not possible since $z\left(s_{0}\right) \neq 0$ by the minimum phase assumption.

(b) For every $\varepsilon^{*}>0$ there exists $\varepsilon \in\left[0, \varepsilon^{*}\right)$ such that $A_{\varepsilon}$ has distinct eigenvalues in $\overline{\mathbb{C}_{+}}$. Suppose this is not the case. Then $p_{\varepsilon}(s)$ has eigenvalues of multiplicity $l \geq 2$ for all $\varepsilon \in\left[0, \varepsilon_{1}\right), \varepsilon_{1}>0$. Therefore

$$
p_{\varepsilon}(s)=(s-\lambda(\varepsilon))^{l} q_{\varepsilon}(s) \text { for all } \varepsilon \in\left[0, \varepsilon_{1}\right)
$$

and in particular it follows from (3.1) that for $s=\lambda(\varepsilon)$

$$
p_{\varepsilon}(s)=(s-\lambda(0))^{\prime} q_{0}(s)+\varepsilon z(s)=0
$$

where $q_{0}(\xi) \neq 0$ in $\left[0, \varepsilon_{1}\right)$. Differentiation w.r.t. $s$ yields also $t \lambda(\varepsilon)$

$p_{\varepsilon}^{\prime}(s)=l(s-\lambda(0))^{(l-1)} q_{0}(s)+(s-\lambda(0))^{l} q_{0}^{\prime}(s)+\varepsilon z^{\prime}(s)=0$ Using $z(\lambda(\varepsilon)) \neq 0$ an algebraic manipulation gives for $s=\lambda(\varepsilon)$

where

$$
(s-\lambda(0))^{(l-1)} \cdot \psi(s) \equiv 0 \quad \text { for } \quad \varepsilon \in\left[0, \varepsilon_{1}\right),
$$

$\psi(s):=l q_{0}(s) z(s)+(s-\lambda(0))\left[q_{0}^{\prime}(s) z(s)-q_{0}(s) z^{\prime}(s)\right]$

Continuity of $\lambda(\varepsilon)$ yields that $\psi(s)$ is uniformly bounded away from 0 for $\varepsilon_{1}$ sufficiently small. Therefore $\lambda(\varepsilon)=\lambda(0)$, which is impossible by (a).

(c) Suppose $A_{0}$ has distinct eigenvalues and for some $\varepsilon_{1}>0, v(\varepsilon) A_{\varepsilon}=\lambda(\varepsilon) v(\varepsilon)$ for $\lambda$ continuous in $\varepsilon \in$ $\left[0, \varepsilon_{1}\right)$. Then there exists an $\varepsilon_{2} \in\left(0, \varepsilon_{1}\right)$ such that

$$
v(\varepsilon) x \neq 0 \text { for all } \varepsilon \in\left(0, \varepsilon_{2}\right) \text {. }
$$

To prove this, it is sufficient to prove the statement for a single $x$ since the unstable subspace is finite dimensional. Note that the eigenvectors depend locally analytically on $\varepsilon$. Therefore it remains to consider the case

$$
v(\varepsilon) x \neq 0 \quad \text { for all } \varepsilon \in\left[0, \varepsilon_{\mathbf{3}}\right) \text { for some } \varepsilon_{\mathbf{3}}>0
$$

This implies that $\left[\begin{array}{cc}\lambda(\varepsilon)-A_{\varepsilon} & x \\ c & 0\end{array}\right]$ is singular whence $\operatorname{det}\left[\begin{array}{cc}\lambda(\varepsilon)-A & x \\ c & 0\end{array}\right]=0$ for all $\varepsilon \in\left[0, \varepsilon_{3}\right)$. By $(\mathrm{a}), \lambda(\varepsilon)$ is not constant. So we have $\operatorname{det}\left[\begin{array}{cc}s I_{n}-A & x \\ c & 0\end{array}\right] \equiv 0$ on $\mathbb{C}$. But this condratics detectability as the initial state $x(0)=x$ for the system $\dot{x}=A x, y=C x$ has zero output.

(d) Now we are able to prove the proposition. Suppose we apply the controller (2.1) and the last switch occurs for $T_{M}$ at time $t=t_{M}$. If the terminal system is not stable replace $T_{M}$ by $T_{M}+\varepsilon$ according to (b) so that the eigenvalues are seperate. Next (c) can be applied to replace $T_{M}$ by $T_{M}+\varepsilon+\varepsilon^{\prime}$ to ensure non-zero projection of $x\left(t_{M}\right)$ into the unstable eigensubspace of $A-\left(T_{M}+\varepsilon+\varepsilon^{\prime}\right) b c$. Repeating this change finitely many times yields exponentially stability of the terminal system. This proves the proposition.

\section{References}

[ 1 ] A. Ilchmann and L. Logemann (1991) High-gain adaptive stabilization of multivariable linear systems - revisited. ; Report No.243, Institut für Dynamische Systeme, Universität Bremen

[ 2 ] A. Ilchmann and D.H. Owens (1991) Exponential stabilization using non-differential gain adaptation; IMA Journal of Math. Control and Inf. 7, 339-349

[ 3 ] T. Kailath (1980) Linear Systems ; PrenticeHall, Englewood Cliffs

[4] R.D. Nussbaum (1983) Some remarks on a conjecture in Parameter Adaptive Control; Systems \& Control Lett. 3, 243-246

[5] J.C. Willems and C.I. Byrnes (1984) Global adaptive stabilization in the absence of information on the sign of the high frequency gain; in: Lect. Notes Control Inf. Sci. 62, Springer-Verlag, Berlin

Acknowledgement

This work was supported, in part, by the German Research Association (DFG) and the EEC SCIENCE programme under grant number ERBSC1 - CT000433. 